\title{
COMPARING THE VALUES OF SERUM HIGH DENSITY LIPOPROTEIN (HDL) LEVEL IN NEONATAL SEPSIS
}

\author{
Ali Aghayar Macooie1, Seyed Hasan Fakhraie², Seyed Abulfazl Afjeh³, Mohammad Kazemian4, Ali Asghar Kolahi ${ }^{5}$ \\ 1 Department of Paediatrics, Faculty of Medicine, Urmia Medical Science University, Urmia, Iran. \\ ${ }^{2}$ Department of Paediatrics, Faculty of Medicine, Shahid Beheshti University of Medical Sciences, Tehran, Iran. \\ ${ }^{3}$ Department of Paediatrics, Faculty of Medicine, Shahid Beheshti University of Medical Sciences, Tehran, Iran. \\ ${ }^{4}$ Department of Paediatrics, Faculty of Medicine, Shahid Beheshti University of Medical Sciences, Tehran, Iran. \\ ${ }^{5}$ Faculty of Medicine, Shahid Beheshti University of Medical Sciences, Tehran, Iran.
}

\begin{abstract}
BACKGROUND
Despite many years of intensive research, attempts to improve the outcome of sepsis and septic shock by modulating the inflammatory response have met with only limited success. This is at least in part a consequence of our limited understanding of the extremely complex mechanisms that regulate the inflammatory response. The development of successful treatments for sepsis and septic shock is, therefore, likely to require further improvements in our understanding of the factors that determine whether an individual responds appropriately to an infectious insult or progresses to uncontrolled systemic inflammation, immune compromise, and organ failure. In recent years, there has been a considerable interest in the ability of high-density lipoproteins (HDLs) to modulate the inflammatory response to sepsis, haemorrhagic shock, and ischemia-reperfusion injury and, hence, in their potential as novel therapeutic agents in these conditions. In this study, our objectives are measurement of normal HDL level, and evaluation of its variation in septic neonates to determine its prognostic and therapeutic values in this group of patients.
\end{abstract}

ABSTRACT

\section{METHODS}

In a descriptive-cross sectional study, 125 normal non-septic neonates and 50 CRP \& blood culture positive septic neonates' serum HDL were analysed and compared. Results were analysed by SPSS Ver. 16 and ANOVA and Tukey tests. There was statistically significant difference in HDL level between normal and septic neonates. This study conducted in Mofid and Mahdiye Educational Hospital of Shahid Beheshti University over a period of 2 years.

\section{RESULTS}

First one hundred normal neonates' HDL was assessed and analysed to determine the normal serum level of HDL. After that, seventy-five neonates in 3 groups: 1- 25 aseptic neonates, 2- 25 septic CRP positive neonates and 3- 25 septic blood culture positive neonates' HDL were assessed and analysed. Three group had no significant difference in general parameters such as gestational age, birth weight, Apgar scoring and many of hematologic and biochemical in parameters. But difference in HDL level, band cell count, CRP level, hospital stay and mortality were statistically significant ( $p$ value $<0.001$ ).

\section{CONCLUSIONS}

HDL decreased in septic neonates and in future it may be used as a new therapeutic modality.

\section{KEY WORDS}

Neonatal Sepsis, Lipopolysaccharide, High Density Lipoprotein, rHDL

HOW TO CITE THIS ARTICLE: Macooie AA, Fakhraie SH, Afjeh SA, et al. Comparing the values of serum high density lipoprotein (HDL) level in neonatal sepsis. J. Evolution Med. Dent. Sci. 2019;8(22):1772-1776, DOI: 10.14260/jemds/2019/389

\section{BACKGROUND}

Bacterial sepsis standard treatment consists of resuscitating and stabilizing the patient's vital signs as well other factors including prescribing the needed water, electrolyte, and calorie; vasopressor factors for improving blood pressure; organs' circulatory and oxygen transport; prescribing antibiotics; and secondary treatments such as IVIG, G-CSF, etc. Nowadays, the main aim of the medical interventions is detecting the process and direction through which the cells

'Financial or Other Competing Interest': None.

Submission 14-04-2019, Peer Review 18-05-2019,

Acceptance 24-05-2019, Published 03-06-2019.

Corresponding Author:

Seyed Hasan Fakhraie,

Department of Pediatrics,

Faculty of Medicine,

Shahid Beheshti University of Medical Sciences,

Tehran, Iran.

E-mail: shfakhraee@aap.net

DOI: $10.14260 /$ jemds $/ 2019 / 389$ responsible for immunity and defending human body are stimulated by bacterial products and this leads to the aggravation of the inflammatory process, more complications, and patients' mortality. However, despite the widespread studies to reduce the complications and mortality arising from sepsis and septic shock through modulating the inflammatory responses, no great success has been achieved. The main factor for this can be our limited knowledge and information about the very complicated mechanisms that regulate inflammatory responses.(1,2,3) In recent years, there has been a considerable interest in the ability of lipoproteins and especially high-density lipoproteins (HDLs) to modulate the inflammatory response to sepsis, haemorrhagic shock, and ischemia-reperfusion injury and, hence, in their potential as novel therapeutic agents in these conditions. $(1,2,4,5)$

With respect to its metabolic behaviours, reconstituted HDL (rHDL) is similar to natural HDL in human and animal models. RHDL can be analysed just like human and animal models, does not bring about immunity reaction, and cannot 
be identified by reticuloendothelial system. Its infusion has been tolerated well in human volunteers and does not disrupt routine tests. Moreover, the recipients of this product will not produce antibody against apoA-I and rHDL. Thus, rHDL is an appropriate substitute for HDL in medical uses. However, it accelerates Candida-albicans growth; it is likely to be dangerous in patients suffering from Candida-albicans. $(6,7)$

Thus, HDL has an important role in the reverse cholesterol transport, and it can be involved in modulating the natural immunity response as well. Some of the enzymes and proteins going along with HDL cause the increase of its anti-inflammatory qualities. SAA is the main apoprotein of HDL in the acute phase that carries HDL and redirect them through detoxification route leading to liver toward macrophage clearance route. The result of this is the rapid HDL interaction as well as low HDL. Low HDL faces the patient with an increased pre-inflammatory stimulation at the time of sepsis and shock. This leads to an impaired cycle consisting of the inflammation arising from LPS, lowered HDL resulting from inflammation, and the decreased LPS neutralization by HDL. This can cause systemic inflammatory response reinforcement, advanced multi-organic injury, and ultimately death. Prescribing rHDL is likely to be effective and safe in preventing, treating, and reactivating the complications of sepsis. However, although this has been proved in animal studies, it needs to be studied and proved in humans as well. Moreover, owing to the structural similarities found between LPS and LTA, it is likely that rHDL is effective and useful in sepsis cases with gram-positive organisms just like sepsis with gram-negative organisms.

In the present study, it was attempted to discuss and define the normal amount of HDL in newborns and its changes resulting from maternal blood lipid profile, delivery factors and events, and different kinds of newborns with respect to pregnancy age, weight, and Intrauterine growth status. It was also attempted to measure the amount of HDL in newborns suffering from sepsis and compare it with the amounts in newborns without any septic problems. In this way, one can determine the diagnostic value and its role in determining the prognosis of newborns suffering from sepsis, and thus open new horizons for a substitute of lowered HDL in newborns suffering from sepsis.

\section{METHODS}

The present study is a descriptive-cross sectional one. It was done to determine the serum HDL mean in newborns in the Education and Treatment Center of Shahid Beheshti University of Medical Sciences in two years from September 2009 to August 2011.

In the first stage, in order to evaluate the mean serum HDL in healthy neonates without clinical and laboratory symptoms of sepsis, serum HDL of 100 neonates (50 female neonates and 50 male neonates) were measured. In the second stage, 3 groups of newborns, including the first group consisting of 50 neonates without clinical and laboratory symptoms of sepsis, the second group consisting of 50 neonates with clinical symptoms of sepsis, positive CRP and negative blood culture, and the third group consisting of 50 neonates with clinical symptoms of sepsis, positive CRP positive and positive blood culture were studied. In these groups, in addition to clinical and laboratory characteristics, serum HDL level was also measured. Accordingly, 50 neonates with final diagnosis sepsis, including 25 neonates with clinical symptoms of sepsis and positive CRP and 25 neonates with clinical symptoms of sepsis and positive CRP and positive blood culture were studied and serum HDL level was measured. Twenty and five neonates without infectious problems, without sepsis clinical symptoms of sepsis and with positive CRP and negative blood culture were used as control group. It should be noted that sampling was performed successively and sepsis in patients was diagnosed with laboratory findings, CRP and positive blood culture in addition to clinical symptoms. It should be noted that the sample size in this study has been considered unequal and proportional, so that the sample size in the control group (normal) was 5 times more than that of other groups (other than normal group), which this sample size calculation was the highest number calculated in the three calculations performed for the study. Accordingly, 125 people were considered for normal group and 25 people were considered for other groups. The information of this calculation was obtained through the pilot test results.

\section{Statistical Analysis}

The data collected was analysed using SPSS-16. The quantitative data was shown as standard deviation and \pm mean, and the qualitative data was shown as frequency (Percentage). Since we are dealing with more than two groups, the test used is ANOVA or one-way analysis of variance. Tukey test was applied to show the relation between one group with any individual groups.

\section{Ethical Considerations}

Before conducting the serologic study, written letter of consent was obtained from the newborns' parents. For conducting the present study no extra sampling or bleeding was done, and the costs of the necessary tests were paid by the administrator of the thesis. The information and data collected remained confidential. The present study was confirmed by the Ethics Committee of Shahid Beheshti University of Medical Sciences.

\section{RESULTS}

25 healthy newborns, 25 newborns with sepsis clinical symptoms and positive CRP and negative blood culture, and 25 newborns with sepsis clinical symptoms and positive CRP and blood culture were studied.

The findings of the tests indicate that the following items did not have a significant statistical difference: pregnancy average age; the percentage of newborn girls/boys; birth weight; first and fifth-minute Apgar score; blood sugar; calcium; sodium; BUN; $\mathrm{Cr}$; the ratio of natural delivery to the Caesarean one; serum haemoglobin; white blood cells count; percentage of neutrophil and Lymphocytes; and platelet count in group 1,2 , and 3.

In group 1, all the patients' CRP was negative; in group 2, it was +2.68 , and in group 3 , it was +2.64 , and this increase was significant in comparison with group 1. Band cell percentage was $0.92,3.48$, and 3.32 in group 1,2 , and 3 respectively; the difference is significant. The duration hospitalization duration was $7 / 56,24 / 56$, and $21 / 28$ in group 1, 2, and 3 respectively; the difference was significant. Mortality percentage of the newborns was 16,24 , and 36 in group 1, 2, and 3 respectively; the percentage of group 1 was 
significantly different from those of group 2 and 3. Serum HDL amounts of all three groups were compared with one another as well as with that of the control group; lowered amount of serum HDL with acceptable statistical difference was indicated between positive blood culture CRP group and control group. In the primary studies, HDL serum level mean was 44.96 (41.96-45.96); it was 40.83 (38.40-45.97) in group $1,29.40$ (25.35-33.45) in group 2, and 28.44 (31.49-25.39) in group 3; this indicates an acceptable statistical difference with $P$ value $<0.001$ between healthy newborns group and control group with two other groups i.e., newborns with Sepsis and newborns with positive CRP and blood culture.

\section{DISCUSSION}

In recent years, there has been a considerable interest in the ability of lipoproteins and especially high-density lipoproteins (HDLs) to modulate the inflammatory response to sepsis, haemorrhagic shock, and ischemia-reperfusion injury and, hence, in their potential as novel therapeutic agents in these conditions. $(1,2,4,5)$

According to the present sources, normal HDL serum level is $50-60 \mathrm{mg} / \mathrm{dl}$ in women, and $40-50 \mathrm{mg} / \mathrm{dl}$ in men. The main reasons for high HDL levels are hyperalphalipoproteinaemia, acute liver diseases, and weight loss. The main reasons for lowered HDL levels include: recent disease; hunger and stress; being overweight; uncontrolled diabetes; Hepatocellular disease; cholestasis, chronic kidney disease; metabolic syndrome (Insulin and hypertriglyceridemia resistance); malnutrition; indolent lifestyle; smoking, familial Abetalipoproteinemia; betablockers usage; thiazide diuretics; steroids; and increased level of immunoglobulins. ${ }^{(8)}$

In the main part of the present study, assuming that HDL serum level is low in newborns suffering from bacterial sepsis with positive blood culture in comparison with newborns without any systemic septic disease,

25 newborns entered our study having exercised the inclusion/exclusion criteria of the present study. They all had sepsis clinical as well as laboratory symptoms and enjoyed positive blood culture. Their general data was recorded, and their HDL serum levels were measure. Moreover, since cases of positive blood culture in newborns suffering from sepsis are rare according to clinical and laboratory evidence such as CRP (Which is a common problem of most treatment centers), 25 newborns suffering from sepsis according to clinical and laboratory evidence with positive CRP and negative blood culture were studied in another group. Alongside the two abovementioned groups, and despite the primary studies on 100 healthy newborns for determining normal HDL serum level in Iranian newborns, 25 newborns, having the general features like the two abovementioned groups, with no septic disease were added as the control group. The serum HDL amounts of the newborns in the three groups were measured. They were then compared with one another and with those of control group. Reduced serum HDL amount with an acceptable statistical difference was observed between positive blood culture group and positive CRP group with that of the control group. The mean of HDL serum level mean was $44.96(41.96-45.96)$ in the primary studies; it was 40.83 (38.40-45.97) in group 1, 29.40 (25.33$33.45)$ in group 2 , and 28.44 (25.39-31.49) in group 3 . The statistical comparison was performed, and the data collected has been shown in the table 1 .

\begin{tabular}{|c|c|c|c|c|c|}
\hline \multicolumn{7}{|c|}{ HNOVA } \\
\hline & $\begin{array}{c}\text { Sum of } \\
\text { Squares }\end{array}$ & Df & $\begin{array}{c}\text { Mean } \\
\text { Square }\end{array}$ & F & Sig. \\
\hline & 7557.977 & 3 & 2519.326 & 29.629 & .000 \\
\hline Between Groups & 14540.000 & 171 & 85.029 & & \\
\hline Within Groups & $\mathbf{2 2 0 9 7 . 9 7 7}$ & $\mathbf{1 7 4}$ & & & \\
\hline Total & Table 1 \\
\hline \multicolumn{7}{|c|}{} \\
\hline
\end{tabular}

\begin{tabular}{|c|c|c|c|c|c|c|c|c|}
\hline \multicolumn{9}{|c|}{ HDL } \\
\hline \multirow[b]{2}{*}{ ڤ్ } & \multirow[b]{2}{*}{$z$} & \multirow[b]{2}{*}{$\underset{\Sigma}{\mathbb{\Xi}}$} & \multirow{2}{*}{ 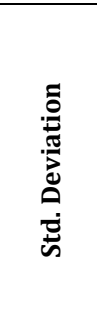 } & \multirow{2}{*}{ 它 } & \multicolumn{2}{|c|}{$\begin{array}{c}95 \% \\
\text { Confidence } \\
\text { Interval for } \\
\text { Mean }\end{array}$} & \multirow{2}{*}{$\stackrel{\Xi}{\Xi}$} & \multirow{2}{*}{ 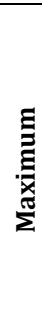 } \\
\hline & & & & & 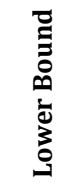 & 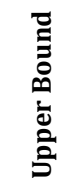 & & \\
\hline Normal & 100 & 44.96 & 10.094 & 1.009 & 41.96 & 45.96 & 22 & 68 \\
\hline 1 & 25 & 38.40 & 5.895 & 1.179 & 35.97 & 40.83 & 23 & 51 \\
\hline 2 & 25 & 29.40 & 9.815 & 1.963 & 25.35 & 33.45 & 11 & 49 \\
\hline 3 & 25 & 28.44 & 7.377 & 1.475 & 25.39 & 31.49 & 18 & 43 \\
\hline Total & 175 & 38.87 & 11.269 & .852 & 37.19 & 40.55 & 11 & 68 \\
\hline \multicolumn{9}{|c|}{ Tab } \\
\hline \multicolumn{9}{|c|}{ Table 2} \\
\hline
\end{tabular}

\begin{tabular}{|c|c|c|c|c|c|c|}
\hline \multicolumn{7}{|c|}{ Multiple Comparisons } \\
\hline \multicolumn{7}{|c|}{ Dependent Variable: HDL } \\
\hline \multicolumn{7}{|c|}{ Tukey HSD } \\
\hline & & Mean & \begin{tabular}{|c} 
Standard \\
Deviation \\
SD
\end{tabular} & $\begin{array}{c}\mathbf{P} \\
\text { value }\end{array}$ & & \\
\hline \multirow{2}{*}{$\begin{array}{c}\text { (I) } \\
\text { Group }\end{array}$} & \multirow{2}{*}{$\begin{array}{c}\text { (J) } \\
\text { Group }\end{array}$} & \multirow{2}{*}{$\begin{array}{c}\text { Mean } \\
\text { Difference } \\
\text { (I-J) }\end{array}$} & \multirow{2}{*}{$\begin{array}{c}\text { Std. } \\
\text { Error }\end{array}$} & \multirow[t]{2}{*}{ Sig. } & \multicolumn{2}{|c|}{$\begin{array}{c}95 \% \\
\text { Confidence } \\
\text { Interval }\end{array}$} \\
\hline & & & & & $\begin{array}{l}\text { Lower } \\
\text { Bound }\end{array}$ & $\begin{array}{l}\text { Upper } \\
\text { Bound }\end{array}$ \\
\hline \multirow{3}{*}{ Normal } & 1 & $5.6^{*}$ & 2.1 & .038 & .21 & 10.91 \\
\hline & 2 & $14.6^{*}$ & 2.1 & .000 & 9.21 & 19.91 \\
\hline & 3 & $15.5^{*}$ & 2.1 & .000 & 10.17 & 20.87 \\
\hline \multirow{3}{*}{1} & Normal & $-5.6^{*}$ & 2.1 & .038 & -10.91 & -.21 \\
\hline & 2 & $9.0^{*}$ & 2.7 & .004 & 2.23 & 15.77 \\
\hline & 3 & $9.9^{*}$ & 2.7 & .001 & 3.19 & 16.73 \\
\hline \multirow{3}{*}{2} & Normal & $-14.6^{*}$ & 2.1 & .000 & -19.91 & -9.21 \\
\hline & 1 & $-9.0^{*}$ & 2.7 & .004 & -15.77 & -2.23 \\
\hline & 3 & .9 & 2.7 & .983 & -5.81 & 7.73 \\
\hline \multirow{3}{*}{3} & Normal & $-15.5^{*}$ & 2.1 & .000 & -20.87 & -10.17 \\
\hline & 1 & $-9.9^{*}$ & 2.7 & .001 & -16.73 & -3.19 \\
\hline & 2 & -.9 & 2.7 & .983 & -7.73 & 5.81 \\
\hline \multicolumn{7}{|c|}{ Table 3} \\
\hline
\end{tabular}

Since we have more than two groups, the test applied is ANOVA or one-way analysis of variance is used in the present study. This test indicates that the groups are significantly different from one another ( $\mathrm{P}$ value $<0.001)$. However, since the difference between the groups is similar, Tukey statistic is use to show the relation between one group with any individual groups.

In the study done by Jung-Yien Chien et al on 63 patients suffering from sepsis, the duration of hospitalization, suffering from hospital infection, and mortality were like the present study. In their study, patients with HDL and apolipoproteins A-I was 20 and under $100 \mathrm{mg} / \mathrm{dl}$ which was more in comparison with patients with normal HDL amounts and apolipoproteins A-I with high statistical values.(9) In a study done by Clementine et al on newborns with sepsis or 
septic shock and dead newborns, the amount of serum cholesterol especially HDL was low at the onset of the decrease in all three groups. However, it was much lower in newborns with septic shock or dead newborns (just like our study), and newborns suffering from sepsis had normal cholesterol when they recovered and discharged in the future days.(10) Yildiz et al studied the diagnostic value of lipids and serum lipoproteins in newborns with late onset sepsis (LOS) on 36 newborns (15 preterm newborns and 21 term newborns), and they used 36 newborns as the control group. Total cholesterol amount, triglycerides, -a lipoprotein, HDL, $A$ and B apolipoproteins were significantly lower in NLOS in comparison with the control group $(p=0.001)$. They concluded that it is likely that HDL function as a useful index for diagnosing NLOS.(11)

In the study done by Molina et al on 135 healthy newborns and newborns with intrauterine growth restriction (IUGR), there was no statistical difference with respect to HDL amount.(12) Narinder et al studied and reviewed numerous researches on the effect of various factors on the amount of triglyceride and serum cholesterol in newborns. Generally speaking, the newborn's umbilical cord cholesterol is lower than normal amounts in adults (especially HDL). Although various factors such as pregnancy age, low birth weight, race, maternal diet, and kind of delivery affect the amount of the newborn's serum lipids, most of these effects are on the level of serum triglyceride than newborn's serum cholesterol.(13) In the study done by Pecks et al, newborns suffering from intrauterine growth restriction (IUGR) had less HDL-c than naturally small newborns, and they are more likely to suffer from coronary atherosclerotic diseases.(14) In the study done by WANG et al on healthy preterm newborns and newborns suffering from RDS, the amount of blood lipids and its different kinds including HDL and LDL did not show much difference, and the difference resulted from the newborn's different weights and ages. (15) In the study done by Elzbieta Pac, the amount of cholesterol and its different kinds were not significant based on the newborn's sex and birth weight.(16) In the study done by Zohreh Badi'ei et al on 378 full-term newborns to determine the serum triglyceride level and different kinds of cholesterol, HDL amount was +31.1/$9.9 \mathrm{mg} / \mathrm{dl}$ in newborn girls and $+28.8 /-8.7 \mathrm{mg} / \mathrm{dl}$ in newborn boys. In this study the total amount of cholesterol and HDL in newborn girls were significantly more than those of the newborn boys.(17) In the study done by Juarez et al on 200 newborns, the amount of serum HDL was $+52.7 /-14 \mathrm{mg} / \mathrm{dl}$. Having studied the abovementioned studies, one can conclude that the average HDL serum level in newborns is around $39.2 \mathrm{mg} / \mathrm{dl}$. Moreover, although various factors including maternal blood lipids status, maternal diet, pregnancy age, fetal intrauterine growth status, and delivery kind affect the serum total cholesterol, the changes affected HDL much less and they did not have high statistical value.

\section{CONCLUSIONS}

HDL direct prescription or factors leading to its increase such as statin have been focused on preventing hypercholesterolemia and exact control of blood sugar with insulin (for using extra cholesterol). Statin can increase HDL, and it has anti-inflammatory effects and modulates the immune system as well (53). Thus, HDL plays an important role in reversing cholesterol transport, and it is possibly involved in modulating the natural immunity responses.

Some of the enzymes and proteins going along with HDL cause the increase of its anti-inflammatory qualities. SAA is the main apoprotein of HDL in the acute phase that carries HDL and redirects them through detoxification route leading to liver toward macrophage clearance route. The result of this is the rapid HDL interaction as well as low HDL. Low HDL in a patient causes an increased pre-inflammatory stimulation at the time of sepsis and shock. This leads to an impaired cycle consisting of the inflammation arising from LPS, lowered HDL resulting from inflammation, and the decreased LPS neutralization by HDL. This can cause systemic inflammatory response reinforcement, advanced multi-organic injury, and ultimately death. Prescribing rHDL is likely to be effective and safe in preventing, treating, and reactivating the complications of sepsis. However, although this has been proved in animal studies, it needs to be studied and proved in humans as well. Moreover, owing to the structural similarities found between LPS and LTA, it is likely that rHDL is effective and useful in sepsis cases with gram-positive organisms just like sepsis with gram-negative organisms.

\author{
Abbreviations \\ VLDL- Very Low-Density Lipoprotein \\ IDL- Intermediate-Density Lipoprotein \\ LDL- Low-Density Lipoprotein \\ HDL- High-Density Lipoprotein \\ LPS - Lipopolysaccharide \\ rHDL- Reconstituted HDL \\ LTA- Lipoteichoic Acid \\ PepG- Peptidoglycan \\ TNF- $\alpha$ - Tumour Necrosis Factor- $\alpha$ \\ NOS- Nitric Oxide Synthase \\ ABCA1- ATP Binding Cassette A1 Transporter \\ RCT- Reverse Cholesterol Transport \\ LPS- LPS Binding Protein \\ SAA- Serum Amyloid A \\ PON1- Human Serum Paraoxonase -1 \\ PAF-AH- Platelet-Activating Factor Acetylhydrolase \\ LPC- Lysophosphatidylcholine \\ PAF- Platelet-activating factor \\ PROWESS- human activated protein $\mathrm{C}$ worldwide evaluation \\ in severe sepsis
}

\section{REFERENCES}

[1] Wu A, Hinds CJ, Thiemermann C. High-density lipoproteins in sepsis and septic shock: metabolism, actions and therapeutic applications. Shock 2004;21(3):210-21.

[2] Rosenson RS, Freeman MW, Saperia GM, et al. Lipoprotein classification, metabolism and role in atherosclerosis. Up To Date: June 11, 2009.

[3] Riedemann NC, Ward PA. Anti-inflammatory strategies for the treatment of sepsis. Expert Opin Biol Ther 2003;3(2):339-50.

[4] Van Leeuwen HJ, van Beek AP, Dallinga-Thie GM, et al. The role of high density lipoprotein in sepsis. Neth J Med 2001;59(3):102-10.

[5] Pajkrt D, Doran JE, Koster F, et al. Anti-inflammatory effects of reconstituted high-density lipoprotein during human endotoxemia. J Exp Med 1996;184(5):1601-8. 
[6] Netea MG, Curfs JH, Demacker PN, et al. Infusion of lipoproteins into volunteers enhances the growth of Candida albicans. Clin Infect Dis 1999;28(5):1148-51.

[7] Williamson MA, Snyder LM, Wallach JB. Wallach's interpretation of diagnostic tests. $9^{\text {th }}$ edn. Philadelphia, USA: Wolters Kluwer Health/Lippincott Williams \& Wilkins 2011.

[8] Chien JY, Jerng JS, Yu CJ, et al. Low serum level of highdensity lipoprotein cholesterol is a poor prognostic factor for severe sepsis. Crit Care Med 2005;33(8):1688-93.

[9] Vermont CL, Den Brinker M, Kakeci N, et al. Serum lipids and disease severity in children with severe meningococcal sepsis. Ped Crit Care Med 2005;33(7):1610-5.

[10] Yildiz B, Ucar B, Aksit A, et al. Diagnostic values of lipid and lipoprotein levels in late onset neonatal sepsis. Scand J Infect Dis 2009;41(4):263-7.

[11] Molina M, Casanueva V, Cid X, et al. Lipid profile in newborns with intrauterine growth retardation. Rev Med Chil 2000;128(7):741-8.
[12] Bansal N, Cruickshank JK, McElduff P, et al. Cord blood lipoproteins and prenatal influences. Current Opinion in Lipidology 2005;16(4):400-8.

[13] Pecks U, Brieger M, Schiessl B, et al. Maternal and fetal cord blood lipids in intrauterine growth restriction. J Perinat Med 2012;40(3):287-96.

[14] Wang H, Zhang W, Wang J, et al. Relationship between early serum lipid profiles and respiratory distress syndrome in preterm infants. Zhongguo Dang Dai Er Ke Za Zhi (Chin J Contemp Pediatr) 2013;15(8):614-8.

[15] Pac-Kozuchowska E. The concentration of lipid parameters in newborns and in older children. Dev Period Med (Med Wieku Rozwoj) 2013;17(1):53-63.

[16] Badiee Z, Kelishadi R. Cord blood lipid profile in a population of Iranian term newborns. Pediatr Cardiol 2008;29(3):574-9.

[17] Juárez IE, Rivera-Silva G, Mejía-Arangure JM, et al. Lipid profile in healthy newborn infants and its correlation with maternal lipid levels. Salud Publica Mex 1999;41(5):405-9. 